\title{
DAS NEUE ASSOZIIERUNGSABKOMMEN VON JAUNDE ${ }^{1}$
}

\author{
Von Friedrich-Wilhelm Albrecht
}

Am 29. Juli 1969 wurde in der Hauptstadt der Bundesrepublik Kamerun, Jaunde (Yaoundé), das „Abkommen über die Assoziation zwischen der Europäischen Wirtschaftsgemeinschaft (im folgenden Gemeinschaft) und den mit dieser Gemeinschaft assoziierten afrikanischen. Staaten und Madagaskar" (im folgenden assoziierte Staaten) (im folgenden Abkommen 1969) unterzeichnet, das an die Stelle des gleichnamigen ebenfalls in Jaunde unterzeichneten Abkommens vom 20. Juli $1963^{2}$ (im folgenden Abkommen 1963) treten wird.

Die Verhandlungen, die übrigens nicht, wie dies Artikel 228 Absatz 1 des Vertrages zur Gründung der Europäischen Wirtschaftsgemeinschaft (im folgenden EWGV) vorsieht, von der Kommission der Europäischen Gemeinschaften (im folgenden Kommission) geführt wurden, haben sich lange hingezogen. Die Aufnahme der Verhandlungen hatte der Assoziationsrat des Abkommens 1963 am 23. Juli 1968 in Kinshasa beschlossen, aber erst am 29. Juni 1969 konnte das Abkommen in Luxemburg paraphiert werden.

Dieser Bericht soll den Inhalt des neuen Abkommens 1969 darstellen und die wichtigsten Abweichungen gegenüber dem Abkommen 1963 erkennbar machen.

Diese Abweichungen sind allerdings zu einem großen Teil redaktioneller Art. Bei Unterzeichnung des neuen Abkommens stand das Ende der im EWGV vorgesehenen Übergangszeit bevor. Dieses Ende der Übergangszeit bringt grundsätzlich die endgültige Beseitigung der zwischen den Mitgliedstaaten bestehenden Zölle, mengenmäßigen Beschränkungen und Maßnahmen (einschließlich Abgaben) gleicher Wirkung mit sich (Artikel 13 und $30 \mathrm{EWGV}$ ) und gegenüber dritten Ländern die vollständige Anwendung des Gemeinsamen Zolltarifs und eine gemeinsame Handelspolitik (Artikel 23 Absatz 3 und 113 EWGV). Auch die im Laufe einer gewissen Zeit stufenweise zu verwirklichenden Ziele der Assoziation waren weitgehend erreicht. Es war daher angebracht, etwa an die Stelle von Bestimmungen, die besagen, daß die Mitgliedstaaten ihre Zölle gegenüber den assoziierten Staaten in gleicher Weise wie untereinander schrittweise beseitigen und daß die assoziierten Staaten ihrerseits die Zölle gegenüber den Mitgliedstaaten nach einem besonders festgelegten Rhythmus abbauen, Bestimmungen zu setzen, wonach die Gemeinschaft gegenüber den assoziierten Staaten bzw. die assoziierten Staaten gegenüber der Gemeinschaft keine Zölle anwenden.

Der Bericht über das Abkommen 1969 soll - nach einigen allgemeinen Bemerkungen (I) - zunächst die Vertragsziele (II), dann die Bestimmungen über die Organe der Assoziation (III), die Bestimmungen für den Warenverkehr zwischen der Gemeinschaft und den assoziierten Staaten (IV), die Handelspolitik gegenüber dritten Ländern (V), den Dienstleistungs-, Kapital- und Personenverkehr (VI), die Bestimmungen über die finanzielle und technische Zusammenarbeit (VII) und schließlich die geltenden Übergangsmaßnahmen (VIII) behandeln (Artikel ohne nähere Bestimmung sind die des Abkommens 1969).

\footnotetext{
1 Zum Abkommen von Jaunde vgl. auch Clauss in VRU 1968, S. $453 \mathrm{ff}$. und - aus kamerunischer Sicht - Merkel in VRU 1969, S. 485 f., sowie in diesem Heft Elsner, S. 221, und die Buchbesprechungen, S. 262. Die lateinamerikanischen Bedenken hat Westphalen in VRU 1969, S. $77 \mathrm{ff}$. , vorgetragen.

2 In Kraft getreten am 1. Juni 1964. Amtsblatt der Europäischen Gemeinschaften vom 11. 6. 1964, S. 1431.
} 


\section{Allgemeines}

Das Abkommen 1969 ist ein Vertrag zwischen der Europäischen Wirtschaftsgemeinschaft nebst ihren sechs Mitgliedstaaten einerseits und 18 außereuropäischen Staaten andererseits. Aus dieser Gegenüberstellung ergibt sich, daß die Vorschriften grundsätzlich nicht das Verhältnis zwischen den assoziierten Staaten oder gar zwischen den Mitgliedstaaten, sondern nur das zwischen beiden Gruppen regeln wollen, obwohl alle Beteiligten am Abkommen „Vertragspartei“ sind. Artikel 56 des Assoziierungsabkommens 1969 stellt klar, daß Verträge aller Art zwischen einem oder mehreren Mitgliedstaaten und einem oder mehreren assoziierten Staaten der Anwendung dieses Abkommens nicht entgegenstehen dürfen.

1. Die assoziierten Staaten sind heute völkerrechtlich selbständige Staaten, die aber bei Inkrafttreten des EWGV mit gewissen Mitgliedstaaten besondere Beziehungen unterhalten haben. Sie und die anderen - in Anlage IV des EWGV aufgeführten - Länder und Hoheitsgebiete ${ }^{3}$ sind damals gemäß Artikel 131-136 EWGV und einem dem EWGV als Anlage beigefügten Durchführungsabkommen über die Assoziierung der UUberseeischen Länder und Hoheitsgebiete mit der Gemeinschaft vom 25. März 1957 (im folgenden Abkommen 1957) assoziiert worden. Das Abkommen 1957 galt nur für einen Zeitraum von 5 Jahren. An seine Stelle war danach für die inzwischen selbständig gewordenen Staaten das Abkommen 1963 getreten, während für die anderen überseeischen Länder und Gebiete der Rat der Europäischen Wirtschaftsgemeinschaft die Assoziation gemäß Artikel 136 Absatz 2 durch den am 1. Juni 1964 in Kraft getretenen Beschluß des Rates vom 25. Februar 1964 über die Assoziation der überseeischen Länder und Gebiete mit der Europäischen Wirtschaftsgemeinschaft ${ }^{4}$ (im folgenden Beschluß 1964) regelte. Die materiellen Vorschriften dieses Beschlusses entsprechen weitgehend denen des Abkommens 1963.

Die 18 von den Abkommen 1963 und 1969 betroffenen Staaten sind folgende: Mit ehemals besonderen Beziehungen zu Belgien: Republik Burundi, Republik Rwanda, Demokratische Republik Kongo;

mit ehemals besonderen Beziehungen zu Frankreich: Republik Dahome, Republik Elfenbeinküste, Republik Gabun, Bundesrepublik Kamerun, Republik Kongo, Republik Madagaskar, Islamische Republik Mali, Republik Mauretanien, Republik Niger, Republik Obervolta, Republik Senegal, Republik Togo, Republik Tschad, Zentralafrikanische Repuplik;

mit ehemals besonderen Beziehungen zu Italien: Republik Somalia, früher Somaliland ${ }^{5}$.

3 Nicht zu diesen Ländern und Gebieten gehören die gemäß Artikel 227 Absatz 2 EWGV zu behandelnden vier französischen überseeischen Departements (Départements d'outre-mer: im folgenden $D$. O. M.) Guadeloupe, Guyane, Martinique und Réunion.

4 Amtsblatt der Europäischen Gemeinschaften vom 8. 2. 1964, S. 424.

$5 \mathrm{Vgl}$. dazu die Absichtserklärung über das zur Zeit unter Verwaltung der Italienischen Republik stehende Treuhandgebiet Somaliland, angefügt an die Schlußakte der Regierungskonferenz für den Gemeinsamen Markt und Euratom vom 25. März 1957.

Ahnliche Absichtserklärungen finden sich dort einerseits für Libyen und andererseits für die unabhängigen Länder der Franken-Zone. Zur Assoziierung ist es bisher nur bei Marokko und Tunesien gekommen: Abkommen zur Gründung einer Assoziation zwischen der Europäischen Wirtschaftsgemeinschaft und der Tunesischen Republik vom 28. März 1969 (unterzeichnet in Tunis) in Kraft getreten am 1. September 1969.

Abkommen zur Gründung einer Assoziation zwischen der Europäischen Wirtschaftsgemeinschaft und dem Königreich Marokko vom 31. März 1969 (unterzeichnet in Rabat) in Kraft getreten am 1. September 1969.

Das Problem Algerien (vgl. Artikel 227, Absatz 2, EWGV) ist bisher ungelöst geblieben. 
Der Beschluß 1964 gilt für Saint-Pierre und Miquelon, Komoren-Archipel, Französisch-Somaliland, Neukaledonien und zugehörige Gebiete, die Wallis- und FutunaInseln, Französisch-Polynesien, die australen und antarktischen Gebiete, Surinam und die Niederländischen Antillen ${ }^{6}$.

Dagegen sind das in Anhang IV EWGV genannte Guinea und das dort aufgeführte frühere niederländische Neuguinea nicht mehr mit der EWG assoziiert.

2. Die Assoziation mit den 18 assoziierten Staaten ist nicht abschließend. In Artikel 60 Absatz 3 des Abkommens 1969 ist ausdrücklich der Beitritt anderer Staaten, deren Wirtschaftsstruktur und Produktion mit denen der assoziierten Staaten vergleichbar ist, vorgesehen.

Anläßlich der Fertigstellung des Abkommens 1969 haben die im Rat vereinigten Vertreter der Regierungen der Mitgliedstaaten auch ihre Absichtserklärung vom 1. April 1963 erneuert, in der es wie folgt heißt: Die Mitgliedstaaten . . . erklären sich bereit, im Wege von Verhandlungen mit dritten Ländern, die einen entsprechenden Antrag stellen und hinsichtlich ihrer Wirtschaftsstruktur und Produktion mit den assoziierten Staaten vergleichbar sind, wohlwollend den Abschluß von Abkommen anzustreben, die zu einer der folgenden Lösungen führen können: Beitritt zum Abkommen nach dem Verfahren des Artikels [58] dieses Abkommens, Assoziierungsabkommen mit gegenseitigen Rechten und Pflichten, insbesondere auf dem Gebiet des Warenverkehrs, Handelsabkommen zur Erleichterung und Förderung des Handels zwischen der Gemeinschaft und diesen Ländern?

3. Beachtung verdient, daß die 6 Mitgliedstaaten der EWG neben der Gemeinschaft die Abkommen 1963 und 1969 unterzeichnet haben ${ }^{3}$. Eine Mitwirkung der Mitgliedstaaten beim Abschluß dieses Assoziierungsabkommens kann ja nur erforderlich sein, soweit sein Inhalt über den durch den EWGV gesteckten Rahmen hinausgeht; innerhalb dieses Rahmens verpflichten die von der Gemeinschaft abgeschlossenen Abkommen nicht nur die Gemeinschaft, sondern auch die Mitgliedstaaten (Artikel 228 Absatz 2 EWGV). Die Assoziierung der in Anhang IV EWGV genannten Länder und Hoheitsgebiete, „um den Handel zu steigern und die wirtschaftliche und soziale Entwicklung durch gemeinsame Beziehungen zu fördern“, wird nun ausdrücklich unter den Tätigkeiten der Gemeinschaft in Artikel 3 Buchstabe k) EWGV genannt, und in Artikel $238 \mathrm{EWGV}$ ist allgemein der Abschluß von Assoziierungsabkommen mit dritten Staaten durch die Gemeinschaft ohne Beteiligung der Mitgliedstaaten vorgesehen.

Selbst wenn anerkannt werden muß, daß finanzielle Hilfe, wie sie in den Abkommen 1957, 1963 und 1969 vorgesehen ist, im allgemeinen nicht Gegenstand von Assoziationen allein nach Artikel 238 EWGV - d. h. ohne Mitwirkung der Mitgliedstaaten - sein kann, so löst das die Frage nicht. Einmal sind auch die auf Artikel 238 gestützten Assoziierungsverträge mit Nigeria ${ }^{9}$ und den ostafrikani-

\footnotetext{
6 Vgl. das zwischen den Mitgliedstaaten abgeschlossene Abkommen über die Anderung des Vertrages zur Gründung der Europäischen Wirtschaftsgemeinschaft mit dem Ziel, daß die in Teil IV dieses Vertrages festgelegte Assoziationsregelung auf die Niederländischen Antillen Anwendung findet vom 13. November 1962; Amtsblatt der Europäischen Gemeinschaften vom 1. Oktober 1964, S. 2415. Vgl. dazu Absichtserklärung im Hinblick a uf die Assoziierung Surinams und der Niederländischen Antillen mit der Europäischen Wirtschaftsgemeinschaft (angefügt an die Schlußakte vom 25. März 1957) und ferner Erklärung in Anhang IX des Assoziationsbeschlusses 1964.

7 Anlage zur Antwort auf die schriftliche Anfrage Nr. 81/63 (Schuijt) Amtsblatt der Europäischen Gemeinschaften vom 11. 12. 1963, S. 2865.

8 Das Abkommen 1957 wurde vor Inkrafttreten des EWGV und daher von den Mitgliedstaaten allein geschlossen.

9 Abkommen zur Gründung einer Assoziation zwischen der Europäischen Wirtschaftsgemeinschaft und der Republik Nigeria vom 16. Juli 1966. Dieses in Lagos unterzeichnete Abkommen, das nach seinem Artikel 32 Absatz 1 nur bis zum 31. Mai 1969 gelten sollte, ist nie in Kraft getreten.
} 
schen Staaten Kenia, Tanzania und Uganda ${ }^{10}$, die keinerlei finanzielle Verpflichtungen enthalten, gemischte Verträge, also Verträge, an denen neben der Gemeinschaft die Mitgliedstaaten Vertragsparteien sind. Zum anderen aber ist im Verhältnis zu den in Anhang IV EWGV genannten Ländern und Hoheitsgebieten in Artikel 132 EWGV, der nicht wie das Assoziierungsabkommen 1957 auf fünf Jahre begrenzt ist, ausdrücklich als Zweck der Assoziation die Beteiligung der Mitgliedstaaten an den Investitionen die Rede, welche die fortschreitende Entwicklung dieser Länder und Hoheitsgebiete erfordert ${ }^{11}$.

Es ist übrigens zweifelhaft, ob und wieweit die Artikel 3 Buchstabe k), 131 bis 135 und 136 EWGV noch im Hinblick auf die inzwischen selbständig gewordenen Staaten gelten. Diese vor Abschluß des Abkommens 1963 sehr umstrittene Frage, die sich für Artikel 3 Buchstabe k), für die Artikel 131 bis 135 und für Artikel 136 nicht notwendig in der gleichen Weise stellt, findet im Abkommen 1963 keine Antwort ${ }^{12}$. Zwar heißt es in Titel und Erwägungsgründen, daß das Abkommen mit den assoziierten, d. h. schon vorher assoziierten Staaten abgeschlossen wird und daß es den Willen bekräftigt, die Assoziation aufrechtzuerhalten. Im übrigen wird aber lediglich der EWGV angerufen, ohne daß bestimmte Vorschriften, etwa Artikel 3 Buchstabe k), die Artikel 131 bis 135, Artikel 136 oder Artikel 238 genannt werden. Das Abkommen 1969 hat diese "Lösung“ übernommen.

4. Das Abkommen 1969 wird am ersten Tage des Monats in Kraft treten, der auf den Tag folgt, an dem die Ratifikationsurkunden der Mitgliedstaaten und die Ratifikationsurkunden von mindestens 15 assoziierten Staaten sowie die Akte zur Notifizierung des Abschlusses des Abkommens durch die Gemeinschaft ${ }^{13}$ beim Sekretariat des Rates der Europäischen Gemeinschaften hinterlegt worden sind (Artikel 57).

Eine nachträgliche Ratifikation durch die anderen assoziierten Staaten ist grundsätzlich nur binnen zwölf Monaten nach Inkrafttreten möglich.

Die Geltungsdauer des Assoziierungsabkommens ist auf fünf Jahre, höchstens aber bis zum 31. Januar 1975 begrenzt (Artikel 59). Die Begrenzung bis zum 31. Januar 1975 soll verhindern, daß die finanzielle Beihilfe der Gemeinschaft aus dem Abkommen von 1969 sich auf einen zu langen Zeitraum erstreckt ${ }^{14}$.

18 Monate vor Ablauf der Geltungsdauer prüfen die Vertragsparteien die Bestimmungen, die für einen weiteren Zeitraum vorgesehen werden können. Es mag bezweifelt werden, ob diese Verlängerung der Frist auf 18 Monate gegenüber 12 Monaten im Abkommen 1963 zur Folge haben wird, daß das dann vereinbarte neue Abkommen rechtzeitig mit Ablauf des Abkommens 1969 in Kraft treten kann. Gegebenenfalls erforderliche Übergangsmaßnahmen bis zum Inkrafttreten eines neuen Abkommens kann der Assoziationsrat treffen (Artikel 60). Das Abkommen

10 Abkommen zur Gründung einer Assoziation zwischen der Europäischen Wirtschaftsgemeinschaft und der Vereinigten Republik Tanzania, der Republik Uganda und der Republik Kenia vom 26. Juli 1968. Auch dieses - in Arusha unterzeichnete - Abkommen ist nie in Kraft getreten und sollte am 31. Mai 1969 ablaufen (Artikel 34 Absatz 1). Am 24. September 1969 wurde in Arusha ein neues gleichnamiges Assoziierungsabkommen unterzeichnet, das noch nicht in Kraft getreten ist.

11 Der vom Rat der Europäischen Wirtschaftsgemeinschaft allein gefaßte Beschluß 1964, der ebenfallı

12 Auch bei einer Bejahung der Fragen dürfte daraus ein eigenes Recht der assoziierten Staaten gegenüber der Gemeinschaft auf Assoziierung bzw. Fortsetzung der Assoziation nicht abzuleiten sein.

13 Der einer Ratifikation durch einen. Staat entsprechende Akt der Gemeinschaft ist der „Abschluß des Vertrages" durch den Rat der Europäischen Gemeinschaft gemäß Artikel 228 EWGV.

$14 \mathrm{Vgl}$. Zur Übergangsregelung, unten S. 219. 
kann von der Gemeinschaft gegenüber jedem assoziierten Staat und von jedem assoziierten Staat gegenüber der Gemeinschaft mit einer Frist von 6 Monaten gekündigt werden (Artikel 62) ${ }^{15}$.

5. Der räumliche Geltungsbereich des Assoziierungsabkommens 1969 ist auf die Hoheitsgebiete der assoziierten Staaten und die europäischen Hoheitsgebiete der Mitgliedstaaten beschränkt (Artikel 55). Nach einer Erklärung der deutschen Bundesregierung (Anhang XIV zur Schlußakte vom 29. Juli 1969) gilt das Assoziierungsabkommen auch für das Land Berlin, sofern sie nicht binnen drei Monaten nach Inkrafttreten des Abkommens gegenüber den übrigen Vertragsparteien eine gegenteilige Erklärung abgibt ${ }^{16}$.

Die Bestimmungen des Titels I des Abkommens (Warenverkehr) gelten auch für die vier französischen D. O. M.17, also für den Handel zwischen ihnen und den assoziierten Staaten.

\section{Ziele des Abkommens}

Es erscheint wichtig, darauf hinzuweisen, daß das Abkommen 1969 - wie das vorhergehende - in Abwendung von kolonialen Strukturen sich in der Präambel ausdrücklich zur Zusammenarbeit auf der Grundlage völliger Gleichberechtigung der Vertragsparteien sowie zur Wahrung der Grundsätze der Vereinten Nationen bekennt.

In die gleiche Richtung weist es, wenn als Ziele der Assoziation besonders die Diversifizierung der Wirtschaft der assoziierten Staaten, ihre Industrialisierung, die Festigung ihres wirtschaftlichen Gleichgewichts und vor allem die Stärkung ihrer wirtschaftlichen Unabhängigkeit genannt werden (Präambel und Artikel 1).

\section{Organe der Assoziation}

Die Assoziation zwischen der Gemeinschaft und den 18 Staaten hat folgende Organe:

- einen Assoziationsrat, der von einem Assoziationsausschuß unterstützt wird,

- eine Parlarnentarische Konferenz,

- ein Schiedsgericht.

1. Der Assoziationsrat besteht aus sechs Mitgliedern des Rates der Europäischen Gemeinschaften — d. h. einem Regierungsmitglied je Mitgliedstaat —, Mitgliedern (in beliebiger Zahl) der Kommission und je einem Mitglied der Regierungen der assoziierten Staaten (Artikel 42). Dagegen ist nicht wie in zwei früheren Assoziierungsabkommen ${ }^{18}$ außerdem je ein Vertreter jedes Mitgliedstaates vorgesehen; praktisch bestünde in diesem Fall ja doch Identität zwischen den Mitgliedern des Rates der Europäischen Gemeinschaften und den Vertretern der Mitgliedstaaten. Den Vorsitz übernimmt abwechselnd der Vorsitzende des Rates der Europäischen Gemeinschaften und ein Mitglied der Regierung eines assoziierten Staates (Artikel 43).

15 Hier könnte es eine Rolle spielen, daß in der Präambel die freundschaftlichen Beziehungen als Grundlage der Zusammenarbeit genannt werden.

16 Vgl. entsprechende Erklärung für den EWGV in der Schlußakte vom 25. März 1957.

17 Vgl. Artikel 227 EWGV.

18 Abkommen zur Gründung einer Assoziation zwischen der Europäischen Wirtschaftsgemeinschaft und Griechenland vom 9. Juli 1961 (unterzeichnet in Athen), in Kraft getreten am 1. November 1962, und Abkommen zur Gründung einer Assoziation zwischen der Europäischen Wirtschaftsgemeinschaft und der Türkei vom 12. September 1963 (unterzeichnet in Ankara), in Kraft getreten am 1. Dezember 1964. 
Der Assoziationsrat tritt mindestens einmal jährlich zusammen. Akte des Assoziationsrates kommen nur einstimmig zustande ${ }^{19}$. Wie jede Seite, d. h. die Gemeinschaft und die assoziierten Staaten, ihre Haltung festlegt, bleibt ihr überlassen ${ }^{20}$ (Artikel 45).

Beschlüsse ${ }^{21}$, die für alle Vertragsparteien verbindlich sind, kann der Assoziationsrat nur in den Fällen treffen, in denen dies ausdrücklich im Abkommen vorgesehen ist (Artikel 46). Diese Beschlüsse sind nicht „self executing“; vielmehr müssen die Vertragsparteien die erforderlichen Durchführungsmaßnahmen treffen. Entschließungen, Empfehlungen und Stellungnahmen bezüglich der Verwirklichung der gemeinsamen Ziele und des einwandfreien Funktionierens der Assoziation sind dagegen immer möglich. Die in einzelnen Bestimmungen des Abkommens 1969 vorgesehenen Konsultationen finden ebenfalls im Assoziationsrat statt. $\mathrm{Zu}$ bedauern ist es, daß Rechtsakte des Assoziationsrates im Amtsblatt der Europäischen Gemeinschaften nicht einmal als „Mitteilung “ veröffentlicht werden.

Aufgaben und Zuständigkeiten des Assoziationsausschusses (Artikel 47), der die für das einwandfreie Funktionieren der Assoziation erforderliche $d$ a u e r n d e Zusammenarbeit gewährleisten soll, legt der Assoziationsrat im einzelnen fest. Dieser Assoziationsausschuß besteht aus je einem Vertreter der sechs Mitgliedstaaten, der Kommission und der 18 assoziierten Staaten. Im übrigen gilt für den Assoziationsausschuß das für den Assoziationsrat Gesagte entsprechend. Die Sekretariatsgeschäfte vom Assoziationsrat und Assoziationsausschuß werden auf paritätischer Grundlage wahrgenommen.

2. Der Zusammenarbeit zwischen dem Europäischen Parlament und den Parlamenten der assoziierten Staaten dient eine Parlamentarische Konferenz ${ }^{22}$, die paritätisch aus Mitgliedern dieser Gremien zusammengesetzt ist, und die Entschließungen verabschieden kann. Der Assoziationsrat legt ihr jährlich einen Tätigkeitsbericht vor (Artikel 52).

3. Streitfälle über Auslegung und Anwendung des Abkommens können, falls eine gütliche Beilegung im Assoziationsrat ${ }^{23}$ nicht $\mathrm{zu}$ erzielen ist, beim Schiedsgericht der Assoziation anhängig gemacht werden (Artikel 53). Allerdings ist das Schiedsgericht des Abkommens 1963 niemals angerufen worden.

\section{Warenverkehr zwischen der Gemeinschaft und den assoziierten Staaten}

Für den Warenverkehr zwischen der Gemeinschaft und den assoziierten Staaten, dessen Erweiterung eines der Hauptziele der Assoziation ist, bestimmt das Abkommen 1969 das Verbot der Zölle und mengenmäßigen Beschränkungen.

Damit läßt sich die aus der Assoziierung fließende gegenseitige Vorzugsbehandlung im Verhältnis zu Drittländern dem GATT gegenüber als eine Freihandelszone rechtfertigen (vgl. Art. XXIV GATT).

19 „Der Assoziationsrat äußert sich im gegenseitigen Einvernehmen der Gemeinschaft einerseits und der assoziierten Staaten andererseits. ${ }^{*}$

20 Vgl. das in Jaunde unterzeichnete Interne Abkommen über die zur Durchführung des Abkommens über die Assoziation zwischen der Europäischen Wirtschaftsgemeinschaft und den mit dieser Gemeinschaft assoziierten afrikanischen Staaten und Madagaskar zu treffenden Maßnahmen und die dabei anzuwendenden Verfahren vom 29. Juli 1969 (im folgenden Internes Durchführungsabkommen 1969). Für die Frage, ob ein Abkommen $\mathrm{zwischen} \mathrm{den} \mathrm{Mitgliedstaaten} \mathrm{notwendig} \mathrm{war} \mathrm{oder} \mathrm{ob} \mathrm{ein} \mathrm{Akt} \mathrm{der} \mathrm{Gemeinschaft} \mathrm{genügt}$ hätte, gilt weitgehend oben S. 203 und 204 Gesagtes.

21 Der im EWGV gebrauchte technische Ausdruck "Entscheidung (Artikel $189 \mathrm{EWGV}$ ) wurde vermieden.

22 Zur Parlamentarischen Konferenz, vgl. Elsner in diesem Heft S. 221.

23 Im Anhang VIII zur Schlußakte des Abkommens 1969 vom 29. Juli 1969 haben sich die Vertragsparteien zu einem Vermittlungsverfahren noch vor Befassung des Assoziationsrates dann bereit erklärt, wenn die Umstände es gestatten. 
Die Vorschriften über den Warenverkehr erfassen auch nur, wie für Freihandelszonen typisch, wenngleich nicht wesentlich, die Waren mit Ursprung in den Vertragsparteien. Die Ursprungsbestimmung mußte daher geregelt werden. Nach Artikel 10 des Abkommens 1969 bleiben die vom Assoziationsrat seinerzeit für die Dauer des Abkommens 1963 erlassenen Vorschriften anwendbar; der Assoziationsrat kann sie jedoch ändern.

Die Assoziation zwischen der Gemeinschaft und den assoziierten Staaten stellt allerdings nicht eine, sondern 18 Freihandelszonen dar. Erfaßt wird nämlich - nach den meisten Bestimmungen ausdrücklich - allein der Handel zwischen der Gemeinschaft und den assoziierten Staaten, dagegen nicht der zwischen den 18 assoziierten Staaten untereinander ${ }^{24}$. Dies war bis żum Inkrafttreten des Abkommens 1963 anders; es ist heute noch anders geregelt in dem Beschluß 1964, der auch den Handel zwischen den überseeischen Ländern und Gebieten erfaßt.

Artikel 5, der sich mit der steuerlichen Behandlung eingeführter Waren beschäftigt, spricht zwar allgemein von Erzeugnissen, die ihren Ursprung in anderen Vertragsparteien haben. Wörtlich genommen gilt diese Vorschrift also auch für den Warenverkehr zwischen den assoziierten Staaten - und übrigens auch zwischen den Mitgliedstaaten. Es erscheint aber mehr als zweifelhaft, daß man Artikel 5 diesen Sinn geben kann ${ }^{25}$.

Das Assoziierungsabkommen ist nur von der Europäischen Wirtschaftsgemeinschaft abgeschlossen, nicht dagegen von der Europäischen Gemeinschaft für Kohle und Stahl und der Europäischen Atomgemeinschaft. Die Vorschriften des Abkommens über den Warenverkehr gelten aber auch für die in Artikel 92 ff. des Vertrages zur Gründung der Europäischen Atomgemeinschaft genannten Erzeugnisse (Anhang $X$ der Schlußakte vom 29. Juli 1969). Ein besonderes Abkommen über die Erzeugnisse, die unter die Zuständigkeit der Europäischen Gemeinschaft für Kohle und Stahl fallen, ist am gleichen Tag wie das Abkommen 1969 in Jaunde zwischen den Mitgliedstaaten und den 18 assoziierten Staaten unterzeichnet worden ${ }^{26}$ und soll gleichzeitig mit diesem Abkommen in Kraft treten.

\section{A. Grundsätze des Warenverkehrs zwischen den Vertragsparteien}

1. E in $\mathrm{fuhrz}$ ölle und Abgaben gleicher Wirkung sind sowohl für die Gemeinschaft (Artikel 2) wie die assoziierten Staaten (Artikel 3) verboten. Im Unterschied zu dem Abkommen 1963 ist eine schrittweise Beseitigung nicht mehr vorgesehen, vielmehr das schlichte Verbot ${ }^{27}$.

Der Begriff der „Abgabe gleicher Wirkung wie Zölle“ dürfte grundsätzlich genauso auszulegen sein wie der gleichlautende Begriff des EWGV, aus dem er stammt (Artikel 12 ff. EWGV). Damit ist allerdings nicht gesagt, daß sich im Rahmen der Assoziation nicht eine Sonderentwicklung ergeben könnte, was allerdings nach Möglichkeit vermieden werden sollte. Zunächst einmal muß jedenfalls die Rechtsprechung des Gerichtshofs der Europäischen Gemeinschaften ${ }^{23}$ herangezogen wer-

\footnotetext{
24 Zollunionen oder Freihandelszonen zwischen den assoziierten Staaten können sich natürlich aus anderen Verträgen ergeben.

$25 \mathrm{Vgl}$. oben S. 202.

$26 \mathrm{Vgl}$. das gleichnamige in Jaunde unterzeichnete Abkommen vom 29. Juli 1963.

$27 \mathrm{Vgl}$. oben S. 201.

$28 \mathrm{Vgl}$. insbesondere die Grundsatzentscheidung in den verbundenen Rechtssachen 2 und $3 / 62$ vom 14. Dezember 1962 (Sammlung der Entscheidungen des Gerichtshofs der Europäischen Gemeinschaften, Band VIII, Seite 867 ff.).
} 
den. Danach ist - vereinfacht gesagt - jede Abgabe, die die Einfuhr belastet, die vergleichbaren inländischen Erzeugnisse jedoch nicht, als zollgleiche Abgabe anzusehen. Auch landwirtschaftliche Abschöpfungen sind daher an sich Abgaben gleicher Wirkung wie Zölle; für die Gemeinschaft ist dies aber ohne Bedeutung, da das Verbot der Abgaben gleicher Wirkung wie Zölle für die einer Abschöpfungsregelung unterliegenden landwirtschaftlichen Erzeugnisse nicht gilt ${ }^{29}$.

Das Verbot der Zölle und Abgaben gleicher Wirkung wird ergänzt durch den schon genannten Artikel 5, der alle Maßnahmen und Praktiken interner steuerlicher Art verbietet, die eine unterschiedliche Behandlung inländischer und gleichartiger eingeführter Erzeugnisse bewirken. Damit ist also auch eine Höherbelastung der Einfuhr gegenüber der Belastung der einheimischen Erzeugung verboten.

Artikel 5 entspricht Artikel 95 Absatz 1 EWGV, der jedoch anders gefaßt ist. Sachliche Unterschiede dürften sich aus dieser anderen Redaktion nicht ergeben. $\mathrm{Ob}$ Artikel 5 auch den in Artikel 95 Absatz 2 EWGV geregelten Tatbestand - auf eine eingeführte Ware wird eine inländische Abgabe erhoben, die geeignet ist, andere inländische Produktionen mittelbar zu schützen - umfaßt, muß bezweifelt werden.

Obwohl die assoziierten Staaten ein dahingehendes Verbot ausdrücklich gewünscht hatten, ist die Erhebung von Verbrauchssteuern an sich, auch auf tropische Erzeugnisse, im Abkommen 1969 nicht verboten worden.

2. Mengenmäßige E in $\mathrm{fuhrbeschränkungen}$ und Maßnahmen gleicher Wirkung sind sowohl für die Gemeinschaft (Artikel 6) wie die assoziierten Staaten (Artikel 7) verboten. Auch hier wird nicht mehr von einer schrittweisen Beseitigung gesprochen $^{30}$. Das Abkommen 1963 erwähnt übrigens bei den Verpflichtungen der Gemeinschaft die Maßnahmen gleicher Wirkung wie mengenmäßige Beschränkungen nicht ${ }^{31}$.

Die Begriffe, die dem EWGV entnommen sind, müssen grundsätzlich wie dort ausgelegt werden. Der Gerichtshof der Europäischen Gemeinschaften hat sich allerdings mit der Definition der „Maßnahmen gleicher Wirkung wie mengenmäßige Einfuhrbeschränkungen" noch nicht befaßt. Nach Auffassung der Kommission sind als solche „Maßnahmen gleicher Wirkung“ jedoch alle Rechts- und Verwaltungsvorschriften und Verwaltungspraktiken anzusehen, welche die Einfuhr oder den Absatz eingeführter Erzeugnisse unmöglich machen oder gegenüber dem Absatz einheimischer Erzeugnisse erschweren bzw. verteuern ${ }^{32}$. Bei dieser weiten Begriffsbestimmung ist eine besondere Vorschrift über Diskriminierungen in den internen Absatzbedingungen - entsprechend Artikel 5 für die internen steuerlichen Diskriminierungen - nicht erforderlich. Auch der EWGV enthält ja im Unterschied zum GATT $^{33}$ keine ergänzenden Vorschriften dieser Art. Dagegen enthält Artikel 7 Absatz 4 - wie der EWGV in Artikel 37 - eine Vorschrift über staatliche Monopole. Die assoziierten Staaten, in denen die Einfuhr einer Ware unter die Zuständigkeit eines staatlichen Handelsmonopols oder einer öffentlichen Stelle fällt, durch die die Einfuhr begrenzt wird, müssen alle Maßnahmen ergreifen, die zur Errei-

29 Vgl. dazu das Protokoll Nr. 12 des Assoziierungsabkommens mit Griechenland, wo es u. a. heißt: „Das im Rahmen der gemeinsamen Agrarpolitik vorgesehene Abschöpfungssystem stellt eine besondere Maßnahme dieser Politik dar, die bei ihrer Anwendung durch eine der.Vertragsparteien nicht als Abgabe mit gleicher Wrrkung wie Zölle im Sinne der Artikel 12 und 37 des Assoziierungsabkommens gilt.*

$30 \mathrm{Vgl}$. oben S. 201.

31 Anders das Abkommen 1957, das in Artikel 10 auf das Kapitel des EWGV über die Beseitigung der mengenmäßigen Beschränkungen zwischen den Mitgliedstaaten schlechthin verweist.

$32 \mathrm{Vgl}$. Antworten auf die schriftlichen Anfragen Nr. 118/66 und 64/67 (Deringer): Amtsblatt der Europäischen Gemeinschaften vom 29. März 1967, S. 901, und vom 26. Juli 1967, Nr. $169 / 11$.

33 Artikel III GATT. 
chung der Ziele der Vorschriften des Abkommens über den Warenverkehr, insbesondere der Gleichbehandlung der Mitgliedstaaten, erforderlich sind. Ob die Auslegungsgrundsätze zu Artikel 37 EWGV hier anweń́bar sind, erscheint wegen der stark abweichenden Fassung der Bestimmungen sehr zweifelhaft. Für die Gemeinschaft fehlt eine entsprechende Verpflichtung, woraus man schließen kann, daß für die betroffenen Waren die allgemeinen Vorschriften (z. B. Verbot der Zölle) gelten.

3. Ergänzt werden die genannten Vorschriften durch die Meis tbegünsti g u n g sk la u s el (Artikel 11 erster Gedankenstrich), nach der — abgesehen vom kleinen Grenzverkehr - die Regelung, welche die assoziierten Staaten auf die Erzeugnisse der Gemeinschaft anwenden, nicht ungünstiger sein darf, als die Regelung für Erzeugnisse mit Ursprung in dem meistbegünstigten dritten Staat.

Zugunsten der assoziierten Staaten fehlt es an einer Meistbegünstigungsklausel. Die Erklärung dafür dürfte darin liegen, daß einmal alle Mitgliedstaaten als Vertragsparteien des GATT zur allgemeinen Meistbegünstigung verpflichtet sind - die außerdem von der Gemeinschaft autonom auch auf Nichtmitglieder des GATT erstreckt wird - und daß andererseits die Gemeinschaft sich nicht zu einer weitergehenden Meistbegünstigung verpflichten konnte, soweit es sich nämlich um Vorteile handelt, die sie nur bestimmten, besonders den anderen mit ihr assoziierten Ländern gewährt, die ja im Verhältnis zwischen der Gemeinschaft und den 18 hier betroffenen Staaten „Drittstaaten“ sind.

4. A u sfuhrzölle und mengenmäßige A usfuhrbeschränkungen sind nach dem Assoziierungsabkommen nicht verboten. Für die Gemeinschaft dürften sie praktisch keine Rolle spielen.

Die assoziierten Staaten sind allerdings verpflichtet, Ausfuhrzölle so zu handhaben, daß sie nicht $z u$ einer unterschiedlichen Behandlung der Mitgliedstaaten führen (Artikel 4). Diese Bestimmungen - wie manches ähnliche Verbot der Diskriminierung zwischen den einzelnen Mitgliedstaaten ${ }^{34}$ - erklären sich daraus, daß ursprünglich jeder assoziierte Staat denjenigen Mitgliedstaat, mit dem er besondere Beziehungen unterhielt, besser stellte als andere Staaten, einschließlich der anderen Mitgliedstaaten.

Die Meistbegünstigungsverpflichtung der assoziierten Staaten bezieht sich auch auf die Ausfuhr (Artikel 11, zweiter Gedankenstrich).

\section{B. Ausnahmen von den Grundsätzen}

Die genannten Verbote und Gebote für den Warenverkehr gelten nicht uneingeschränkt. Das Abkommen sieht vielmehr eine Reihe von Einschränkungen und Sonderregelungen vor.

1. Alle Vertragsparteien können sich auf zwei Ausnahmen berufen.

Einmal dürfen entsprechend Artikel 36 EWGV Verbote und Beschränkungen angewendet werden, die aus Gründen der öffentlichen Sittlichkeit, Ordnung und Sicherheit notwendig sind, oder zum Schutze der Gesundheit und des Lebens von Menschen, Tieren oder Pflanzen, des nationalen Kulturguts von künstlerischem, geschichtlichem oder archäologischem Wert oder des gewerblichen und kommerziellen Eigentums (Artikel 9). 34 Artikel 3 Absatz 3; Artikel 7 Absatz 3; Artikel 7 Absatz 4 des Abkommens; Artikel 3 des Protokolls sind übrigens nicht aufgestellt worden. 
Ferner können die erforderlichen Schutzmaßnahmen ergriffen werden, wenn in einem Wirtschaftsbereich einer Vertragspartei ernste Störungen auftreten oder wenn ihre äußere finanzielle Stabilität durch ernste Störungen beeinträchtigt wird oder auch wenn Schwierigkeiten auftauchen, welche die wirtschaftliche Lage eines Gebietes in den Vertragsparteien verschlechtern (Artikel 16).

Diese Maßnahmen können auch von der Gemeinschaft als solcher gegenüber den assoziierten Staaten angewendet werden; es handelt sich also keineswegs nur darum, daß die Rechte, welche die einzelnen Mitgliedstaaten aus Schutzklauseln des EWGV ${ }^{35}$ haben, auf das Verhältnis zu den assoziierten Staaten ausgedehnt werden. Soweit dies mit dem EWGV vereinbar ist, kann allerdings ein Mitgliedstaat allein Schutzmaßnahmen gegenüber den assoziierten Staaten anwenden. Einzelheiten sind im Internen Durchführungsabkommen 1969 festgelegt.

2. Zugunsten der Gemeinschaft ist bei den Einfuhrzöllen, Abgaben gleicher Wirkung, den mengenmäßigen Einfuhrbeschränkungen und Maßnahmen gleicher Wirkung ausdrücklich bestimmt, daß das Verbot nicht solche Maßnahmen betrifft, die die Mitgliedstaaten noch untereinander anwenden. In der Tat bestehen wohl auch noch über das Ende der Übergangszeit des EWGV hinaus gewisse Reste an solchen Maßnahmen, und es wäre unangebracht gewesen, den assoziierten Staaten eine Besserstellung gegenüber anderen Mitgliedstaaten zu gewähren. In dem Abkommen 1963 war dieses Problem dadurch vermieden, daß zugunsten der assoziierten Staaten auf den Abbau dieser Maßnahmen zwischen den Mitgliedstaaten verwiesen wurde. $\mathrm{Ob}$ die Einschränkung auch bei dem Verbot der steuerlichen Diskriminierung in Artikel 5 gilt, könnte bezweifelt werden, da es an einer dahin gehenden ausdrücklichen Bestimmung fehlt.

3. Auch auf dem Gebiet des Warenverkehrs wurde im Abkommen dem Rechnung getragen, daß die 18 Staaten Entwicklungsländer sind. Erst diese Berücksichtigung des Entwicklungsstandes der assoziierten Staaten gibt der Assoziation ihre moralische Berechtigung und entzieht sie dem Vorwurf, ein neo-kolonialistisches System zu sein.

a) Die assoziierten Staaten können Zölle und Abgaben gleicher Wirkung (Artikel 3 Absatz 2) beibehalten und neu einführen, wenn sie den Erfordernissen ihrer Entwicklung entsprechen oder der Finanzierung des Haushalts dienen. Für die zweite Gruppe ist weder erforderlich, daß sie „Finanzzölle“ im Sinne des Artikels 17 EWGV sind, noch daß sie zur Finanzierung des Haushalts notwendig sind. Die assoziierten Staaten sind also keineswegs verpflichtet, ihren Finanzbedarf soweit wie möglich aus inneren Steuern und aus Zöllen gegenüber dritten Ländern zu befriedigen. Andererseits ist die bloße Bezeichnung einer Abgabe als „Finanzabgabe“ (droit fiscal) allein noch kein Beweis ihrer Zulässigkeit. Zölle oder Abgaben können auch zulässig sein, wenn sie beiden $Z$ wecken gleichzeitig dienen.

Desgleichen können die assoziierten Staaten im Hinblick auf die Erfordernisse ihrer Entwicklung oder bei Auftreten von Zahlungsbilanzschwierigkeiten mengenmäßige Einfuhrbeschränkungen oder Maßnahmen gleicher Wirkung anwenden (Artikel 7 Absatz 2). Im Unterschied zum Abkommen 1963 können zur Förderung der Entwicklung nunmehr mengenmäßige Beschränkungen gleichzeitig mit Zollmaßnahmen vorgenommen werden. Vollständige Einfuhrverbote sollen regelmäßig nicht angewandt werden.

35 Z. B. Artikel 108 und 109 EWGV. 
In den Protokollen Nr. 2 und 3 wird das Verfahren für diese Ausnahmen geregelt. Ferner wird der Begriff der Erfordernisse der Entwicklung genauer umrissen. Nach Artikel 1 des Protokolls Nr. 2 ergeben sich diese Erfordernisse aus:

- „der Durchführung der auf die Anhebung des allgemeinen Lebensstandards ihrer Länder ausgerichteten Programme zur wirtschaftlichen Entwicklung;

- den Erfordernissen ihrer wirtschaftlichen Entwicklung, insbesondere hinsichtlich der Schaffung von Produktionszweigen, die zur Anhebung des allgemeinen Lebensstandards ihrer Länder gefördert werden sollten;

- der Notwendigkeit, ihre Zahlungsbilanz auszugleichen, die Schwierigkeiten zu beheben, die hauptsächlich auf die Anstrengungen zur Ausweitung ihres Binnenmarktes zurückzuführen sind, sowie die Unbeständigkeit ihrer Austauschrelation (terms of trade) zu beseitigen;

- dem Erfordernis, die schnelle und anhaltende Zunahme der Ausfuhrerlöse ihrer Länder zu gewährleisten."

b) Die Meistbegünstigungsverpflichtung der assoziierten Staaten ist ebenfalls eingeschränkt, um die Kooperation zwischen den Entwicklungsländern, insbesondere zwischen den assoziierten Staaten zu fördern. In der Präambel und in Artikel 1 ist gerade der Ausbau der Zusammenarbeit und des Handels zwischen den 18 assoziierten Staaten als Ziel der Assoziation genannt ${ }^{36}$. Die Meistbegünstigungsverpflichtung gilt daher nicht für Zollunionen und Freihandelszonen sowie für andere Abkommen über die wirtschaftliche Zusammenarbeit, an denen nur assoziierte Staaten teilnehmen (Artikel 11). Es kann in diesem Fall auch von allen anderen Vorschriften des Abkommens abgewichen werden.

Die Meistbegünstigung braucht ferner dann nicht gewährt zu werden, wenn es sich um Zollunionen, Freihandelszonen oder andere Abkommen über wirtschaftliche Zusammenarbeit mit anderen afrikanischen Staaten von vergleichbarem Entwicklungsstand handelt. Für diesen Fall ist jedoch ausdrücklich bestimmt, daß dann, wenn außer der Meistbegünstigungsklausel noch andere Grundsätze oder Bestimmungen des Abkommens berührt werden, der Assoziationsrat die für das reibungslose Funktionieren der Assoziation erforderlichen Maßnahmen trifft; dem Assoziationsrat scheinen hier also weitgehende Befugnisse gegeben zu sein. Die Vorschriften über die Bestimmung des Ursprungs von Waren dürfen allerdings in gar keinem Fall beeinträchtigt werden. Zollunionen, Freihandelszonen oder Abkommen über wirtschaftliche Zusammenarbeit mit anderen Drittländern sind dagegen nur unter Beachtung aller Vorschriften des Abkommens einschließlich der Meistbegünstigungsvorschrift zulässig. Das Abkommen ist insoweit strenger als das GATT.

Das Abkommen 1963 hatte in Artikel 9 den zweiten und dritten Fall zusammengefaßt, dabei aber nicht klar bestimmt, ob die Meistbegünstigungsklausel eingeschränkt wurde.

4. Eine wichtige Sonderregelung ist für die Einfuhr gewisser Erzeugnisse in die Gemeinschaft vorgesehen (Artikel 2 Absatz 2; Artikel 6 Absatz 2).

a) Es handelt sich einmal um die landwirtschaftlichen Erzeugnisse im Sinne des EWGV $^{37}$, soweit sie einer gemeinsamen Marktorganisation nach Artikel 40 EWGV unterliegen.

36 Vgl. dazu auch Artikel 19 Absatz 2, der im Zusammenhang mit der Finanzhilfe von der Zweckmäßigkeit regionaler Zusammenarbeit spricht.

37 D. h. Erzeugnisse, die in der Liste des Anhangs II EWGV aufgeführt sind. 
b) Die Sonderregelung gilt ferner für Waren, die zwar nicht landwirtschaftliche Erzeugnisse sind, die aber „bei der Einfuhr in die Gemeinschaft wegen der Durchführung der gemeinsamen Agrarpolitik einer Sonderregelung unterliegen". Mit dieser Formel werden Erzeugnisse erfaßt, die eng mit den Marktorganisationen zusammenhängen, wie Waren, die aus landwirtschaftlichen Erzeugnissen gewonnen worden sind - sogenannte Verarbeitungserzeugnisse - oder die mit landwirtschaftlichen Erzeugnissen in Wettbewerb stehen.

Für die genannten Warengruppen gelten die Vorschriften des Artikels 2 über das Verbot der Zölle und Abgaben gleicher Wirkung nur insoweit, als für Erzeugnisse lediglich Einfuhrzölle erhoben werden und im Rahmen der gemeinsamen Agrarpolitik keine anderen Bestimmungen über den Handel mit dritten Ländern (Abschöpfungen, Mindestpreise usw.) vorgesehen sind (Artikel 2 des Protokolls Nr. 1).

Für alle anderen von der Ausnahme erfaßten Erzeugnisse, soweit die assoziierten Staaten daran ein wirtschaftliches Interesse haben, legt die Gemeinschaft für die Dauer des Abkommens eine besondere Einfuhrregelung fest ${ }^{38}$, die günstiger ist als die allgemeine Regelung für die Erzeugnisse aus dritten Ländern. An dieser Stelle des Abkommens wird also eine wirkliche "Präferenz" stipuliert, die es grundsätzlich - ausschließt, allen dritten Ländern das gleiche oder gar ein günstigeres Einfuhrregime zu gewähren. Protokoll Nr. $4^{39}$ dürfte aber im Rahmen eines allgemeinen Präferenzsystems für Entwicklungsländer Ausnahmen zulassen.

Wird eine Marktorganisation der Gemeinschaft geändert, dann kann auch das besondere Einfuhrverfahren geändert werden, wobei jedoch den assoziierten Staaten vergleichbare Vorteile gewährt werden müssen. (Artikel 3 Absatz 2 des Protokolls Nr. 1)

Auf dem Gebiet der mengenmäßigen Beschränkungen und Maßnahmen gleicher Wirkung ist lediglich bestimmt, daß das Verbot in Artikel 6 Absatz 1 der Einfuhrregelung der genannten Warengruppen nicht vorgreift. Die Gemeinschaft ist danach frei, ob und welche Maßnahmen sie gegenüber den assoziierten Staaten anwenden will. Kontingente oder Maßnahmen gleicher Wirkung dürften jedoch tatsächlich nur für verhältnismäßig wenig Waren angewandt werden.

Diese Regelung für die genannten Waren stellt eine Weiterentwicklung gegenüber dem Abkommen 1963 dar. Damals bezog sich die Ausnahme von der Vorschrift der Zölle und mengenmäßigen Beschränkungen nur auf „die den europäischen Erzeugnissen gleichartigen und mit ihnen konkurrierenden Agrarerzeugnisse".

Jetzt, wo die gemeinsame Agrarpolitik weitgehend festgelegt war, konnte die Regelung auf das Einfuhrregime beschränkt werden, wobei etwas genauere Angaben als damals gemacht werden konnten. Der Kreis der betroffenen Erzeugnisse ist allerdings erweitert worden.

5. Für Erdölerzeugnisse hat sich die Gemeinschaft in Anhang II der Schlußakte vom 29. Juli 1969 vorbehalten, bei Einführung einer gemeinsamen Energiepolitik die Regelung des Abkommens - bei Gewährung vergleichbarer Vorteile - zu ändern.

6. Sofern assoziierte Staaten auf Grund internationaler Verpflichtungen, welche sie einer besonderen Zollregelung unterwerfen ${ }^{40}$, sich nicht in der Lage glauben, die Zölle nur gegenüber der Gemeinschaft zu senken, ist die Gemeinschaft — trotz

38 Wenn es die wirtschaftliche Lage in der Gemeinschaft bei einem Erzeugnis rechtfertigt, kann die Gemeinschaft aber ausnahmsweise von einer Sonderregelung absehen.

39 Vgl. unten S. 213.

40 Z. B. Kongoakte vom 26. 2. 1885 R.G.Bl. S. 215. (Vgl. Vertrag von St. Germain-en-Laye rom 10. 9. 1919). 
fehlender Gegenseitigkeit - gehalten, das Assoziierungsabkommen diesen Ländern gegenüber anzuwenden. Eine Überprüfung der Lage ist nach spätestens drei Jahren vorgesehen (Artikel 63).

\section{Regeln für die Handelspolitik gegenüber dritten Ländern}

Sowohl die Gemeinschaft wie auch - unter Berücksichtigung der Meistbegünstigungsklausel - die assoziierten Staaten sind, da es sich nicht um eine Zollunion handelt, in der Gestaltung ihrer Handelspolitik frei.

1. Das Abkommen 1969, wie auch das vorhergehende, enthält mit Ausnahme der erwähnten Regelung nach Protokoll Nr. $1^{41}$ keine Verpflichtung zur Gewährung einer echten Präferenz, d. h. einer Vorzugsbehandlung im Verhältnis zu dritten Ländern.

2. Soweit handelspolitische Maßnahmen die Interessen der anderen Seite beeinträchtigen können, besteht eine gegenseitige Unterrichtungspflicht (Artikel 15). Insbesondere ist die Gemeinschaft verpflichtet, den Assoziationsrat über Anträge auf Beitritt zur Gemeinschaft oder Assoziierung mit ihr zu unterrichten (Artikel 60 Absatz 1). Diese Bestimmung dürfte nicht anwendbar sein, wenn die Anträge nur die Erneuerung bestehender Assoziierungen betrifft; dann könnte aber die allgemeine Klausel des Artikels 15 eingreifen.

Das Protokoll Nr. 5 sieht vor, daß die Vertragsparteien ihre gegenseitigen Interessen auf nationaler Ebene berücksichtigen und zu diesem $Z$ weck zusammenarbeiten und einander unterstützen. Dies gilt insbesondere für die Probleme des Absatzes und der Vermarktung tropischer Erzeugnisse.

Im Hinblick auf die Bemühungen der Konferenz der Vereinten Nationen über Handel und Entwicklung (UNCTAD/CNUCED) um allgemeine Präferenzen zugunsten der Entwicklungsländer stellt das Protokoll Nr. 4 klar, das das Assoziierungsabkommen, insbesondere sein Artikel 3, der Verwirklichung eines allgemeinen Präferenzsystems auch zugunsten der assoziierten Staaten nicht entgegensteht.

Erwähnenswert ist, daß das Abkommen 1969 in der Präambel ganz allgemein auch die Entwicklung der internationalen Wirtschaftsbeziehungen als Ziel bezeichnet. Die Gemeinschaft hat bereits verlauten lassen, daß mit Inkrafttreten des Abkommens 1969 die Sätze des Gemeinsamen Zolltarifs für einige tropische Erzeugnisse - in Form einer Zollsenkung bzw. -aussetzung - herabgesetzt werden sollen.

3. Eine besondere Klausel enthält die Erklärung in Anhang XI der Schlußakte vom 29. Juli 1969, nach der Deutschland eine Aufstockung seines Zollkontingents für Bananen aus dritten Ländern erst nach Konsultierung der assoziierten Staaten vornehmen kann. Dieses Zollkontingent und seine Erhöhung werden durch das Protokoll über das Zollkontingent für die Einfuhr von Bananen vom 25. März 1957 geregelt. 


\section{Niederlassung, Dienstleistung, Zahlungs- und Kapitalverkehr}

Während der Warenverkehr eine eingehende und im Grundsatz die Hemmnisse beseitigende Regelung gefunden hat, gehen die Bestimmungen über das Niederlassungsrecht, den Dienstleistungs-, Zahlungs- und Kapitalverkehr viel weniger weit. Eine Regelung der Freizügigkeit der Arbeitnehmer fehlt überhaupt.

1. Für das Niederlassungsrecht und die Dienstleistungen, die in Artikel 33, 34 und 35 entsprechend den Vorschriften des EWGV ${ }^{42}$ definiert werden, sind lediglich Verpflichtungen der assoziierten Staaten festgelegt; auch diese Verpflichtungen gehen nicht sehr weit: einmal ist eine unterschiedliche Behandlung der Angehörigen und Gesellschaften der Mitgliedstaaten ${ }^{43}$ verboten (Artikel 31), zum anderen gibt es eine Meistbegünstigungsklausel (Artikel 32), die aber ausdrücklich regionale $\mathrm{Ab}-$ kommen ausnimmt.

Das Diskriminierungsverbot $\mathrm{zwischen}$ den Mitgliedstaaten gilt indessen nur insoweit, als der betroffene Mitgliedstaat den Angehörigen und Gesellschaften des betreffenden assoziierten Staates für die in Frage kommende Tätigkeit gleichartige Vergünstigungen gewährt ${ }^{44}$.

2. Was den Kapitalverkehr angeht, so enthält das Assoziierungsabkommen 1969 (Artikel 39) für die assoziierten Staaten das Gebot der Gleichbehandlung der Angehörigen und Gesellschaften der Mitgliedstaaten hinsichtlich der Investitionen und den sich daraus ergebenden Zahlungen. Ferner werden sich die assoziierten Staaten bemühen, für Investitionen und die damit verbundenen laufenden Zahlungen dann eine liberalere Devisenregelung anzuwenden, wenn sie von Personen vorgenommen werden, die in den Mitgliedstaaten ansässig sind.

3. Artikel 37 verpflichtet jeden Unterzeichnerstaat, Zahlungen für den Waren-, Dienstleistungs- und Kapitalverkehr und für Arbeitsentgelte sowie den Transfer dieser Zahlungen in den Mitgliedstaat oder den assoziierten Staat, in dem der Gläubiger oder der Begünstigte ansässig ist, zu genehmigen, soweit der Waren-, Dienstleistungs-, Kapital- und Personenverkehr nach dem Assoziierungsabkommen liberalisiert ist.

In der Tat muß eine Liberalisierung des Warenverkehrs, wie sie im Abkommen geregelt ist, um sinnvoll zu sein, notwendigerweise die Freiheit der entsprechenden Zahlungen umfassen oder durch eine dahingehende besondere Regelung ergänzt werden ${ }^{45}$.

Für das Niederlassungsrecht, die Dienstleistung und den Kapitalverkehr, für die lediglich zugunsten der Gemeinschaft eine Nichtdiskriminierung bzw. die Meistbegünstigung bestimmt sind, dürfte Artikel 37 klarstellen, daß sich diese Gebote auch auf die damit zusammenhängenden Zahlungen erstrecken. Was die Arbeitsentgelte angeht, so kann nur die im Rahmen der Niederlassungsfreiheit erfolgende Beschäftigung von leitenden Kräften gemeint sein, denn von den Arbeitnehmern an sich spricht das Abkommen 1969 nicht.

Darüber hinaus werden sich die Vertragsparteien auch um eine weitergehende Liberalisierung des Zahlungsverkehrs bemühen (Erklärung in Anhang $\mathrm{V}$ der Schlußakte vom 29. Juli 1969).

$42 \mathrm{Vgl}$. Artikel 52, 58 und 60 EWGV.

43 Gemäß einer Erklärung der deutschen Regierung gelten als deutsche Staatsangehörige alle Deutschen im Sinne des Grundgesetzes (Anhang XIII der Schlußakte vom 29. Juli 1969). Vgl. die entsprechende Erklärung in der Schlußakte vom 25. März 1957.

44 Sog. negative Reziprozitätsklausel.

45 Vgl. Artikel 106 Absatz 1 und 2 EWGV. 
Artikel 38 enthält eine Artikel 37 entsprechende Bestimmung für die Zahlungen, die sich aus den Darlehen, Vorschüssen oder Beteiligungen ergeben, die zur Entwicklungshilfe der Gemeinschaft gehören.

\section{Finanzielle und technische Zusammenarbeit}

Der bisher behandelte Inhalt des Abkommens 1969 unterscheidet sich seiner Natur nach nicht wesentlich vom Inhalt der anderen von der Gemeinschaft abgeschlossenen ${ }^{46}$ oder unterzeichneten ${ }^{47}$ Assoziierungsabkommen mit außereuropäischen Ländern ${ }^{48}$.

Den besonderen Aspekt bekommt die Assoziierung mit den 18 afrikanischen Staaten und Madagaskar durch die Finanzhilfe der Gemeinschaft.

Diese Finanzhilfe war bereits Teil der Assoziation seit ihrem Beginn 1958. Artikel $132 \mathrm{EWGV}$ sieht sie ausdrücklich vor. $\mathrm{Zu}$ diesem $Z$ weck wurde gemäß Artikel 1 des Abkommens 1957 der 1. Europäische Entwicklungsfond (im folgenden EEF) gegründet. Durch das Interne Abkommen über die Finanzierung und Verwaltung der Hilfe der Gemeinschaft von 1963 (im folgenden Internes Finanzierungsabkommen 1963) wurde ein 2. EEF eingesetzt und durch das gleichnamige Abkommen vom 29. Juli 1969 (im folgenden Internes Finanzierungsabkommen 1969) ein 3. EEF. Außerdem sah das Abkommen 1963 auch eine Intervention der Europäischen Investitionsbank (im folgenden EIB) vor; das Abkommen 1969 folgt ihm darin.

Die finanzielle und technische Zusammenarbeit ist in Titel II des Abkommens 1969 und dem dazugehörigen Protokoll Nr. 6 über die Verwaltung der Hilfe der Gemeinschaft geregelt. Die Verpflichtung der einzelnen Mitgliedstaaten untereinander, insbesondere über die Aufbringung der Mittel, und das Verfahren zur Prüfung der Anträge sind in dem Internen Finanzierungsabkommen 1969 festgelegt.

\section{A. Höhe und Aufbringung der Hilfe}

Was die Höhe der zur Verfügung gestellten Beträge angeht, so läßt sich gegenüber dem Abkommen 1963 eine Erhöhung feststellen, die allerdings nicht ganz den Wünschen der assoziierten Staaten entspricht.

In den 3. EEF werden für die assoziierten Staaten 828 Millionen Rechnungseinheiten ${ }^{49}$ eingebracht (1963: 666 Millionen Rechnungseinheiten), davon werden 748 Millionen Rechnungseinheiten (1963: 620 Millionen Rechnungseinheiten) in Form nichtrückzahlbarer Zuschüsse und 80 Millionen Rechnungseinheiten (1963: bis zu 46 Millionen Rechnungseinheiten) in Form von Darlehen zu Sonderbedingungen und Beiträgen zur Bildung von haftendem Kapital, insbesondere in Form von Beteiligungen verwendet (Artikel 18 Buchstabe a).

Das Interne Finanzierungsabkommen bezieht sich auch auf die Tätigkeit des EEF in den assoziierten überseeischen Ländern und Gebieten und den französischen D. O. M., denen weitere 72 Millionen Rechnungseinheiten (1963: 64 Millionen Rechnungseinheiten) zukommen sollen.

46 Marokko und Tunesien.

47 Nigeria und Ostafrika.

48 Außer Betracht bleiben müssen in diesem Rahmen Griechenland und die Türkei, deren Assoziation als eine Vorstufe auf Mitgliedschaft in der Gemeinschaft gedacht ist.

49 Eine Rechnungseinheit entspricht dem Goldwert des US \$ (Protololl Nr. 7). 
Der sich daraus ergebende Gesamtbetrag von 900 Millionen Rechnungseinheiten wird von den Mitgliedstaaten wie folgt aufgebracht:

Belgien
Bundesrepublik Deutschland
Frankreich
Italien
Luxemburg
Niederlande

80,0 Millionen Rechnungseinheiten 298,5 Millionen Rechnungseinheiten 298,5 Millionen Rechnungseinheiten 140,6 Millionen Rechnungseinheiten 2,4 Millionen Rechnungseinheiten 80,0 Millionen Rechnungseinheiten

$\mathrm{Zu}$ den Interventionen des 3. EEF tritt noch die EIB, die bis zu 90 Millionen Rechnungseinheiten (1963: 64 Millionen Rechnungseinheiten) Darlehen geben kann (Artikel 18 Buchstabe b) ${ }^{50}$. Der Gesamtbetrag der Hilfe der Gemeinschaft ist daher 1 Milliarde Rechnungseinheiten (1963: 800 Millionen Rechnungseinheiten).

Eine Aufteilung der Hilfe auf einzelne assoziierte Staaten die es für die Produktions- und Diversifizierungshilfe im Abkommen 1963 gegeben hat, ist im neuen Abkommen nicht vorgenommen worden.

Hat ein assoziierter Staat dieses Abkommen nach Maßgabe des Artikels 59 nicht ratifiziert oder ist das Abkommen gemäß Artikel 64 gekündigt worden, so ergibt sich daraus für die Vertragsparteien die Verpflichtung, die Beträge der in diesem Abkommen vorgesehenen Finanzhilfe anzupassen (Artikel 30). Andererseits darf aber durch einen Beitritt weiterer Staaten zum Assoziierungsabkommen die Hilfe an die 18 assoziierten Staaten auch nicht verringert werden (Artikel 60 Absatz 3).

\section{B. Formen der Hilfe}

1. Darlehen zu Sonderbedingungen können für höchstens 40 Jahre gewährt und während der ersten 10 Jahre von der Tilgung befreit werden; die Darlehen der EIB können eine Laufzeit bis zu 25 Jahren haben (Artikel 7 und 8 des Protokolls Nr. 6).

2. Für Darlehen der EIB können aus dem EEF Zinsvergütungen gewährt werden, durch die der tatsächlich zu zahlende Zins bis auf 3 Prozent herabgesetzt werden kann (Artikel 18 Buchstabe b des Abkommens; Artikel 8 des Protokolls Nr. 6). Wird das Darlehen über ein zwischengeschaltetes Organ für die Finanzierung von Entwicklungsmaßnahmen, etwa die Investitionsbank eines assoziierten Staates, durchgeführt ${ }^{51}$, so kann diesem gegenüber der Satz bis auf 2 Prozent sinken. Damit soll erreicht werden, daß der Zins für die Endbegünstigten gegebenenfalls bis auf 3 Prozent gesenkt werden kann. Die Höhe der Zinsvergütung liegt regelmäßig bei 2 oder 3 Prozent. Der Gesamtbetrag der Zinsvergütungen wird auf den Betrag des EEF für nichtrückzahlbare Zuschüsse angerechnet.

3. Eine wesentliche Neuerung des Abkommens 1969 ist die Möglichkeit für die Gemeinschaft - nicht dagegen für die EIB - zur Bildung von haftendem Kapital dadurch beizutragen, daß sie die Eigenmittel von Unternehmen durch Beteiligung oder andere geeignete Mittel verstärkt (Artikel 9 des Protokolls Nr. 6). Diese Beiträge müssen minoritären Charakter haben und sind zeitweiliger Natur; sie

50 Weitere 10 Millionen Rechnungseinheiten (1963: 6 Millionen Rechnungseinheiten) stellt die EIB nach dem Internen Finanzierungsabkommen 1969 für die überseeischen Länder und Gebiete und die französischen D. O. M. zur Verfügung.

51 Sogenannte zweistufige Darlehen, deren Zulässigkeit im Abkommen 1969 zum ersten Mal geregelt wurde. 
sollen zurückgezogen werden, sobald es die Lage des Empfängers erlaubt. Eine Abtretung wird dann vorzugsweise an andere Beteiligte oder doch jedenfalls an Angehörige oder Gesellschaften der assoziierten Staaten erfolgen. Abtretungserlöse und Einkünfte aus Beiträgen werden dem EEF gutgeschrieben.

$\mathrm{Da}$ weder der EEF noch auch die Kommission Rechtspersönlichkeit haben, sondern nur die Gemeinschaft als solche (Artikel 210 EWGV), kann nur diese Beteiligungen erwerben. Es wird notwendig sein, nur solche Beteiligungen $\mathrm{zu}$ wählen, die die Haftung der Gemeinschaft auf bestimmte Beträge begrenzen (Aktien z. B.).

4. Empfänger der Hilfe können nicht nur die assoziierten Staaten selber sein, sondern auch gewisse juristische Personen der Mitgliedstaaten oder der assoziierten Staaten, die nicht in erster Linie einen Erwerbszweck verfolgen, von allgemeinem Interesse sind und staatlicher Aufsicht unterliegen; ferner kommen gewisse Erzeugerverbände, ausnahmsweise auch die Erzeuger selber, sowie regionale oder zwischenstaatliche Einrichtungen in Frage. Gewisse Formen der Hilfe können auch Fachinstituten und selbst Unternehmen, die nach industriellen und kaufmännischen Methoden geführt werden, zugute kommen (Artikel 25).

Die Hilfe der Gemeinschaft kann sowohl als Mitfinanzierung von Vorhaben, an denen andere Geber beteiligt sind, als auch als Finanzierung solcher Vorhaben, an denen neben assoziierten Staaten benachbarte dritte Staaten interessiert sind, erfolgen.

\section{Verwendung der Hilfe}

1. Die zu finanzierenden Vorhaben werden in vier Gruppen eingeteilt, wobei aber ausdrücklich integrierte Vorhaben durch eine aufeinander abgestimmte Anwendung verschiedener Maßnahmen als zweckmäßig angesehen werden (Artikel 19).

a) Die erste Gruppe umfaßt Investitionen auf den Gebieten der Erzeugung und der wirtschaftlichen und sozialen Infrastruktur (vgl. Artikel 1 des Protokolls Nr. 6). Diese Investitionen sollen nach Möglichkeit im Rahmen eines Entwicklungsprogramms oder -plans erfolgen.

Um eine Koordinierung der Arbeit des EEF und der Entwicklungsvorhaben der assoziierten Staaten zu ermöglichen, unterrichten die assoziierten Staaten die Kommission - nach Möglichkeit bei Inkrafttreten des Abkommens - über ihre Entwicklungspläne und -programme und später über alle Änderungen (Artikel 22).

b) Die sogenannte technische Zusammenarbeit geschieht entweder im Zusammenhang mit Investitionen (zweite Gruppe) oder ist allgemeiner Art (dritte Gruppe) (Artikel 2 und 3 des Protokolls Nr. 6).

c) Schließlich wird Verkaufs- und Vermarktungshilfe gewährt (Artikel 4 des Pro-* tokolls Nr. 6).

2. Fortgefallen ist gegenüber dem Abkommen 1963 die Produktionshilfe, obwohl die assoziierten Staaten auf eine Fortsetzung großen Wert gelegt hätten. Die Produktionshilfe, die nicht als dauernde Preisstützung gedacht war, sondern eine schrittweise - z. T. in einem genauen Zeitplan festgelegte - Anpassung an die Erfordernisse einer Vermarktung zu Weltmarktpreisen ermöglichen sollte, hatte nach dem Abkommen 1963 ausdrücklich am Ende des Abkommens auszulaufen.

Der im Abkommen 1963 vorgesehene Reservefonds wurde dagegen wesentlich ausgebaut (Artikel 20). Nunmehr kann aus dem Reservefonds Hilfe geleistet werden zur Unterstützung der assoziierten Staaten bei der Überwindung beson- 
derer und außergewöhnlicher Schwierigkeiten, die zu einer Ausnahmesituation führen, schwerwiegende Auswirkungen auf ihre Wirtschaftspolitik haben und auf einen Sturz der Weltmarktpreise oder auf Ereignisse wie Hungersnot oder Überschwemmungen zurückzuführen sind. Wenn dieser Reservefonds, der nicht mehr wie im Abkommen 1963 auf Naturkatastrophen beschränkt ist, auch kein Ersatz der früheren Produktionshilfe sein soll und kann, so vermag er doch gewisse Folgen aus deren Fortfall zu mildern. Der Reservefonds wird aus den nichtrückzahlbaren Zuschüssen gespeist.

Ferner können zum Ausgleich der Folgen vorübergehender Schwankungen der Weltmarktpreise aus den verfügbaren Kassenbeständen des 3. EEF zinslose Vorschüsse bis zu 50 Millionen Rechnungseinheiten gewährt werden (Artikel 21).

\section{Die Verwaltung der Hilfe}

Der Assoziationsrat legt die allgemeine Ausrichtung der finanziellen und technischen Zusammenarbeit fest (Artikel 29). Die Prüfung der von den assoziierten Staaten vorgelegten Finanzierungsanträge obliegt der Gemeinschaft in Kontakt mit den assoziierten Staaten (Artikel 23).

Der 3. EEF wird nach dem Internen Finanzierungsabkommen 1969 von der Kommission verwaltet, soweit nicht der EIB gewisse Aufgaben übertragen sind. Die Zusammenarbeit zwischen Kommission und EIB wird im einzelnen durch das Interne Finanzierungsabkommen 1969 geregelt.

a) Die Entscheidung über die Finanzierungsanträge an den EEF trifft zwar die Kommission, jedoch werden die Anträge vorher einem EEF-Ausschuß vorgelegt. Dieser Ausschuß setzt sich aus Vertretern der Mitgliedstaaten zusammen, deren Stimmen entsprechend ihrem Anteil an der Finanzierung wie folgt gewogen werden:

$\begin{array}{lr}\text { Belgien } & 9 \\ \text { Bundesrepublik Deutschland } & 33 \\ \text { Frankreich } & 33 \\ \text { Italien } & 15 \\ \text { Luxemburg } & 1 \\ \text { Niederlande } & 9\end{array}$

Der Ausschuß gibt seine Stellungnahme mit einer qualifizierten Mehrheit von 67 Stimmen ab. Ein Mitgliedstaat allein kann also positive Stellungnahmen nicht verhindern.

Beschließt die Kommission, von der Stellungnahme dieses Ausschusses abzuweichen, oder hat der Ausschuß einen Finanzierungsvorschlag nicht befürwortet, so kann sie den Finanzierungsvorschlag dem Rat vorlegen, der mit gleicher Stimmenwägung beschließt.

Eine Bindung der Gemeinschaft an eine Zusage dürfte gegenüber dem Begünstigten erst mit dem Abschluß eines Finanzierungsabkommens nicht schon mit dem Beschluß der Kommission oder des Rates eintreten, selbst wenn er dem betroffenen assoziierten Staat gemäß Artikel 23 mitgeteilt worden ist.

b) Besondere Bestimmungen (Artikel 26) sollen sicherstellen, daß die Unternehmen der Mitgliedstaaten, aber auch der assoziierten Staaten, gleichen Zugang zu den Aufträgen, die mit Mitteln des EEF finanziert werden, erhalten. 
Bei Bauaufträgen begrenzten Umfangs und bei Lieferungsaufträgen können die Bauunternehmen, Industrien und handwerklichen Betriebe der betreffenden assoziierten Staaten oder eines anderen assoziierten Staates des gleichen Gebiets begünstigt werden.

\section{VIII. Übergangsmaßnahmen}

Das Abkommen 1963 ist am 31. Mai 1969 abgelaufen. Jedoch sind seine Bestimmungen durch die Entscheidung des durch dieses Abkommen eingerichteten Assoziationsrates vom 29. Mai 1969 bis zum Inkraftreten eines neuen Abkommens spätestens jedoch bis zum 30. Mai 1970 verlängert worden. Diese Entscheidung stützt sich auf Artikel 60 Absatz 2 des Abkommens 1963, der den Assoziationsrat ausdrücklich ermächtigt, gegebenenfalls die erforderlichen Übergangsmaßnahmen zu treffen.

Auf dem Gebiet der finanziellen Hilfe ist die Übergangsregelung praktisch sehr eingeschränkt, als von den Mitgliedstaaten keine neuen finanziellen Mittel zur Verfügung gestellt worden sind, so daß während der Übergangszeit nur die Hilfe im Rahmen der noch vorhandenen Mittel weitergeführt werden ${ }^{52}$ und die Hilfe aus dem Abkommen von 1969 vorbereitet werden kann.

Auch der Beschluß 1964 ist am 31. Mai 1969 abgelaufen, und an seine Stelle wird ein neuer Beschluß53 des Rates der Europäischen Gemeinschaft treten, der gleichzeitig mit dem Abkommen 1969 in Kraft treten soll. Bis dahin gelten Úbergangsvorschriften, die der Regelung für die assoziierten Staaten entsprechen (Beschluß des Rates vom 28. Mai 1969) ${ }^{54}$.

52 Diese Fortsetzung der Hilfe bis zur Erschöpfung des 2. EEF war übrigens schon in Artikel 3 des Internen Finanzierungsabkommens 1963 vorgesehen.

53 Artikel 136 Absatz 2 EWGV kann nicht nur für einen einzigen neuen Zeitabschnitt gelten, sondern erlaubt auch wiederholte Erneuerungen.

54 Amtsblatt der Europäischen Gemeinschaften vom 31. März 1969, Nr. L 130,64. 